\title{
Der nationale Service für Geodaten: geodata4edu.ch
}

\section{Book Chapter}

Author(s):

Piguet, Arlette

Publication date:

2018

Permanent link:

https://doi.org/10.3929/ethz-b-000283823

Rights / license:

Creative Commons Attribution-NonCommercial-NoDerivatives 4.0 International

Originally published in:

https://doi.org/10.1515/9783110553796-012 


\title{
Arlette Piguet \\ Der nationale Service für Geodaten: geodata4edu.ch
}

\begin{abstract}
Geodaten sind in einer ganzen Reihe von Disziplinen eine äusserst wichtige Grundlage für Wissenschaft, Forschung und Lehre. Der neue nationale Service geodata4edu.ch soll Studierenden und Forschenden in der Schweiz den Zugang zu lizenzpflichtigen Geodaten - zugeschnitten auf die jeweiligen Bedürfnisse - erleichtern. Die Hochschule für Technik Rapperswil (HSR) sowie die ETH Zürich, vertreten durch das Institut für Kartografie und Geoinformation (IKG) und die ETH-Bibliothek, haben geodata4edu.ch in einem gemeinsamen zweijährigen Projekt aufgebaut. Das nutzerorientierte Portal bietet einen zentralen Zugang zu Geodaten von Bundesämtern und Kantonen. Gegenwärtig wird der Service schweizweit in der akademischen Community bekannt gemacht, um einen möglichst breiten Kundenkreis zu gewinnen. Projektgelder stehen im Rahmen des Programms P-5 „Wissenschaftliche Information“ von swissuniversities noch bis Ende 2018 zur Verfügung. Bis dahin soll geodata4edu.ch an den Schweizer Hochschulen etabliert und der Service weiter gestärkt sein.
\end{abstract}

\section{Vorbemerkungen}

Geodaten oder georeferenzierte Daten sind digitale Informationen, denen auf der Erdoberfläche eine bestimmte räumliche Lage zugeordnet werden kann. ${ }^{1}$ Solche Informationen spielen etwa in den Bereichen Fernerkundung und Kartographie, Geomatik, Geographie, Umwelt- und Erdwissenschaften, Raum- und Landschaftsplanung, aber auch in den Bauingenieurwissenschaften und in der Architektur eine grosse Rolle. Auch für soziologische, demographische, politische, touristische und sogar bibliothekarische Fragestellungen sind Geoinformationen ein wichtiges Hilfsmittel.

Angesichts dieser breiten Nutzungsmöglichkeit und der wichtigen Bedeutung von Geodaten stellt sich für die Forschenden, Lehrenden und Studierenden immer wieder die Frage, wo sie die benötigten Daten in der gewünschten Qualität, im geeigneten Datenformat sowie mit dem gewünschten Zeitstand finden können.

1 Weitere Informationen unter: https://www.swisstopo.admin.ch/de/wissen-fakten/geoinforma tion.html. Alle Links in diesem Beitrag wurden am 26.03.2018 überprüft.

2 Open Access. (c) Arlette Piguet, publiziert von De Gruyter. (c) BY-NC-ND Dieses Werk ist lizenziert unter der Creative Commons Attribution-NonCommercial-NoDerivatives 4.0 Lizenz. 
Ist der Bezug der Daten allenfalls kostenpflichtig? Können die Daten visualisiert und für die Weiterverarbeitung auch auf den eigenen Rechner geladen werden?

In der Schweiz existieren verschiedene Dienste und Projekte, in deren Zentrum die Visualisierung und/oder der Zugang zu Geodaten stehen (vgl. hierzu das Kapitel „Das Projekt“). Es kann jedoch festgehalten werden, dass es bisher keine Applikation für Schweizer Hochschulen gab, die es erlaubt, sämtliche, schweizweit flächendeckenden swisstopo-Geodaten ${ }^{2}$ direkt $\mathrm{zu}$ visualisieren, $\mathrm{zu}$ extrahieren, in den von den Benutzenden gewünschten Formaten herunterzuladen und damit den Anforderungen und Kenntnissen entsprechend direkt in Lehre und Forschung einzusetzen. Auch Dienstleistungen für ein umfassendes Angebot an diversen Geodatensets mit Daten von Bund und Kantonen waren in der Schweiz bisher nicht existent. Dies kann u.a. damit begründet werden, dass für den Aufbau einer entsprechenden Dienstleistung die Initiative für die Bündelung der erforderlichen Fachkenntnisse fehlte und auch die finanziellen Mittel für ein entsprechendes Vorhaben nicht vorhanden waren.

Der nationale Service geodata4edu. $\mathrm{ch}^{3}$ soll die skizzierte Lücke im Geodatenangebot für den akademischen Bereich schliessen.

\section{Die Dienstleistung}

Geodata4edu.ch ${ }^{4}$ wurde im Frühjahr 2017 im Rahmen eines Launch-Events einem breiten Fachpublikum sowie den interessierten Hochschulen von den Betreiberinnen des Service vorgestellt. Dies sind die Hochschule für Technik Rapperswil $(\mathrm{HSR})^{5}$ sowie die ETH Zürich, vertreten durch das Institut für Kartografie und Geoinformation (IKG) ${ }^{6}$ und die ETH-Bibliothek ${ }^{7}$. Nach der Veranstaltung wurde die Dienstleistung in eine erste Betriebsphase überführt und die Akquisition der ersten Kundinnen im Schweizerischen akademischen Bereich lanciert.

Geodata4edu.ch bietet über ein benutzerfreundliches Portal zentralen $\mathrm{Zu}$ gang zu einem umfassenden Angebot an ausgewählten lizenzpflichtigen Geodaten von Bundesämtern und Kantonen. Die Schweizer Hochschulen können den Service geodata4edu.ch nutzen, indem sie ,assoziierte Teilnehmer‘ werden.

2 Swisstopo (https://www.swisstopo.admin.ch/): Bundesamt für Landestopographie.

3 Das Projekt startete unter dem Namen „Geodata4SwissEDU“, der produktive Service wurde dann in „geodata4edu.ch“ umbenannt.

$4 \mathrm{https} / /$ www.geodata4edu.ch/.

5 HSR: https://www.hsr.ch/.

6 IKG: http://www.ikg.ethz.ch/.

7 ETH-Bibliothek: http://www.library.ethz.ch/de/. 
Dafür ist einerseits ein Vertrag mit geodata4edu.ch für die Nutzung des Services sowie den Datenbezug erforderlich, andererseits auch Verträge über die geltenden Nutzungsrechte mit den verschiedenen Datenherren. Ein finanzieller Beitrag an den Betrieb der Dienstleistung muss von jeder Hochschule gemäss Businessplan geleistet werden.

Über das zentrale Einstiegsportal www.geodata4edu.ch (vgl. Abb.1) gelangen die Nutzerinnen und Nutzer zum Metadatenkatalog, in dem die vorhandenen Geodaten über das Suchfeld gezielt gesucht werden können. Die Suchbegriffe können Titel eines Datensatzes, Schlagwörter oder Themen umfassen und dreisprachig eingegeben werden (D, F, E). Die Suchdimension „Inhalt“ kann mit den Suchdimensionen „Raum“ und „Zeit“ verknüpft werden.

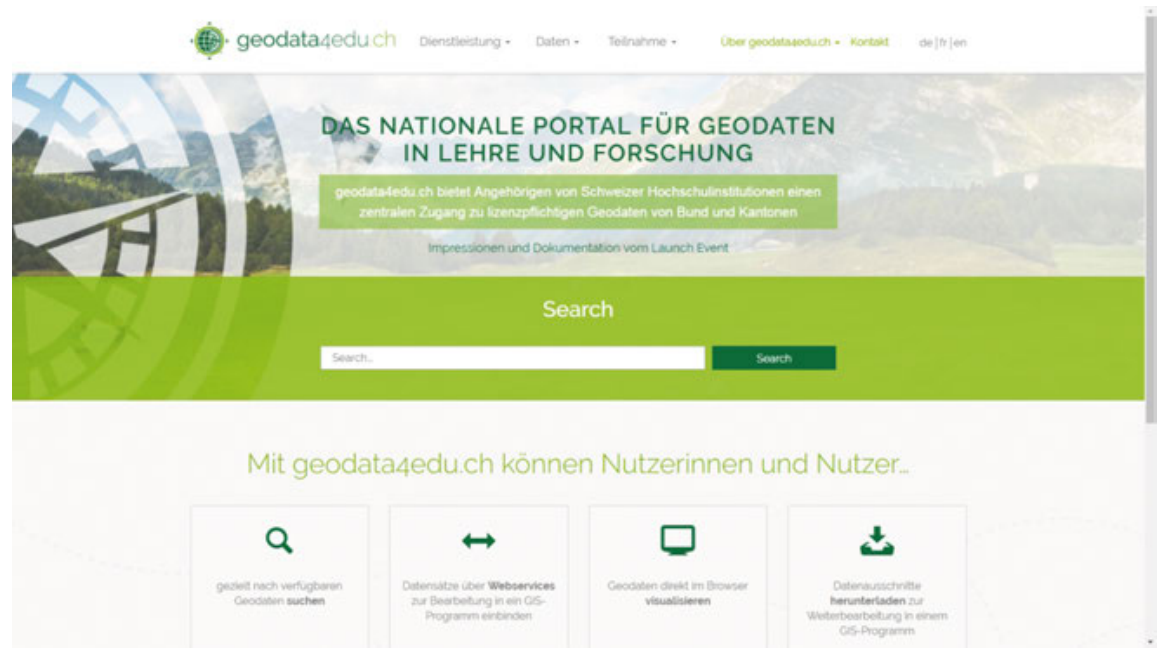

Abb. 1: Portal geodata4edu.ch

Die Ergebnisliste umfasst alle gefundenen Treffer und zeigt diese nach Relevanz geordnet mit den wichtigsten Informationen zum Datensatz an (vgl. Abb. 2), u.a. Titel, Publikationsjahr, Verweis auf den Datenherrn und Servicetyp (vgl. unten). Ein Abstract kann ebenfalls angezeigt werden. Mit einem weiteren Klick gelangt man auf die gesamten einsehbaren Informationen eines Datensatzes, d.h. auch auf die Nutzungsbestimmungen, das Koordinatensystem u.a. Die Metadaten werden in der Sprache angezeigt, in der sie vom Datenherrn oder dem Service geliefert werden (i.d.R. Deutsch oder Englisch). 


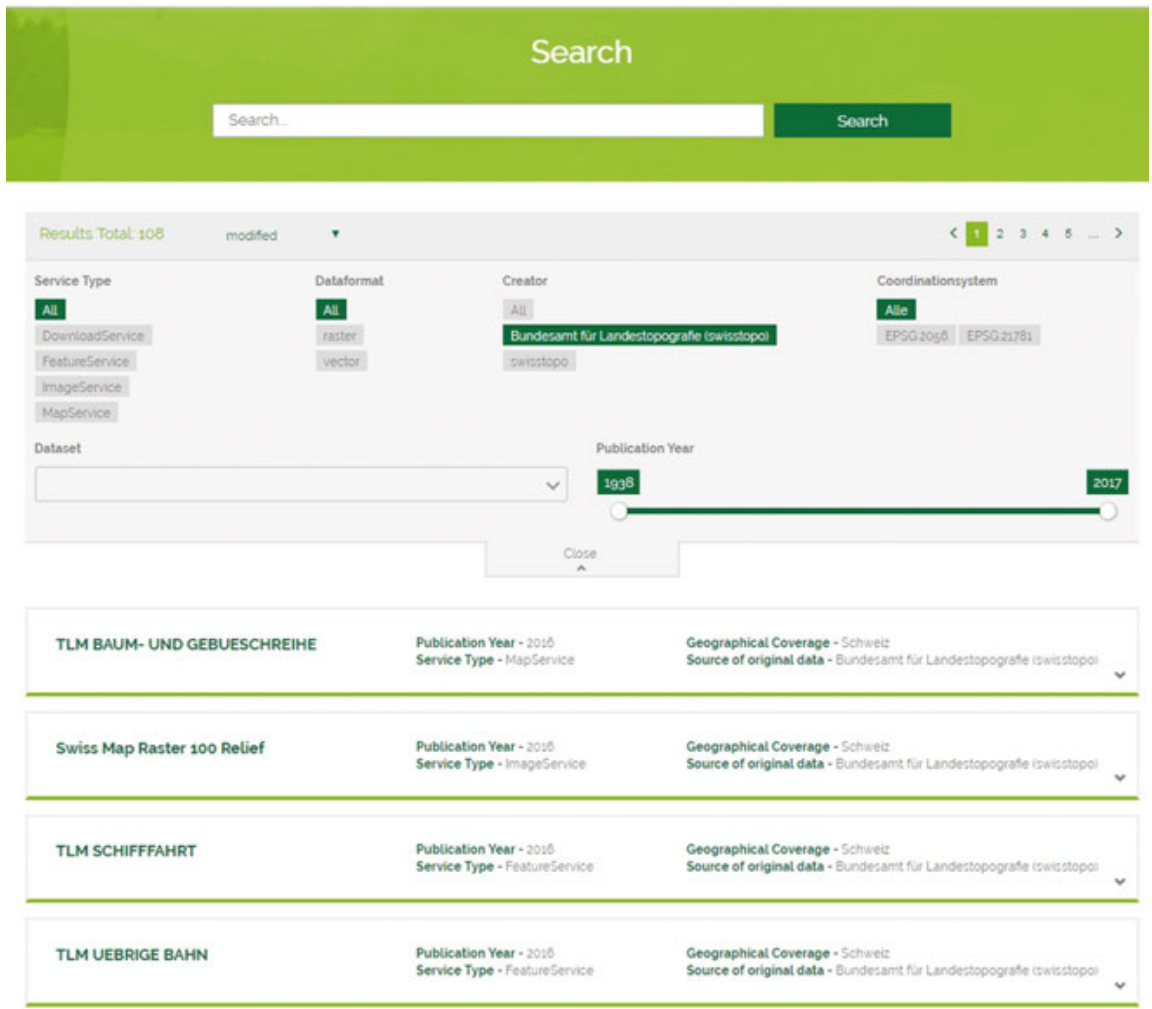

Abb. 2: Ergebnisliste einer Suchanfrage in geodata4edu.ch

Während die Suche im Metadatenkatalog öffentlich zugänglich ist, ist für den Zugriff auf die Geodaten eine Authentifizierung über SWITCHaai nötig. Diese Zugangsberechtigung erhalten die Forschenden, Lehrenden und Studierenden der assoziierten Teilnehmer.

Die zwei unterschiedlichen Zugänge zu den Daten (Servicetypen) werden den heterogenen Anforderungen der Nutzerinnen und Nutzern gerecht: Der Downloadservice, d.h. der webbasierte Zugang zu Datenausschnitten über eine graphische Nutzeroberfläche, eignet sich vor allem für Personen mit wenig GIS-Erfahrung, der Direktzugriff auf ganze Datensätze via Webservices hingegen ist für Personen mit bereits guten GIS-Kenntnissen ausgelegt (vgl. Abb. 3). 


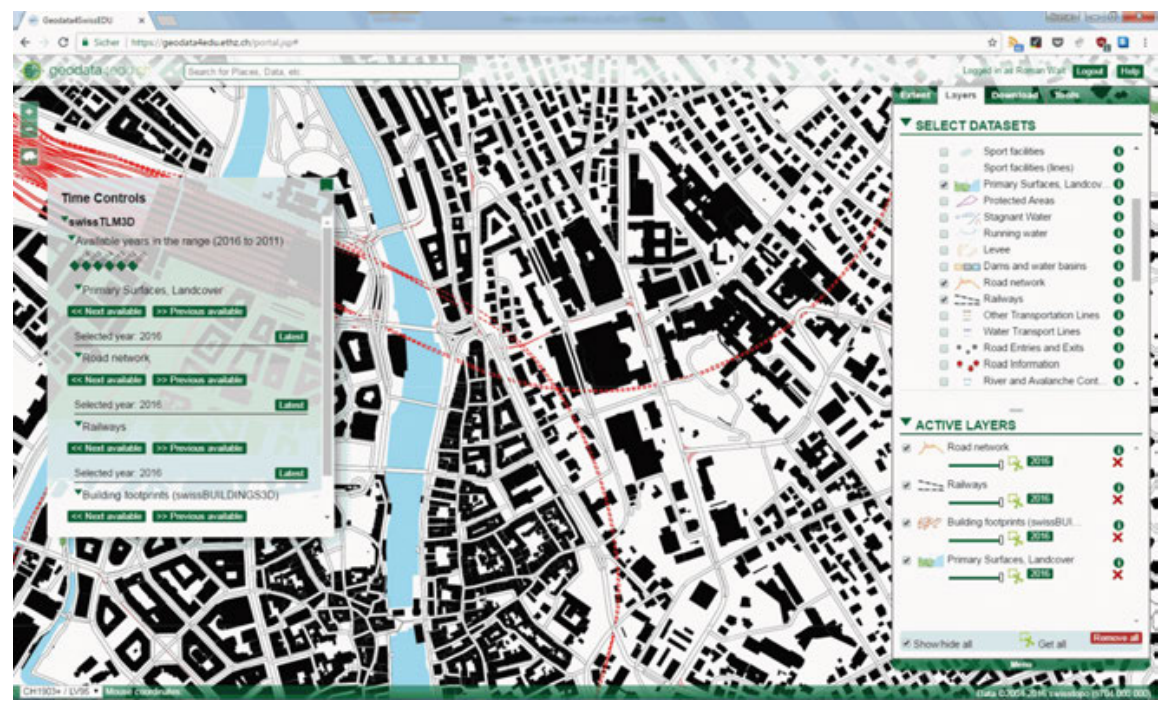

Abb. 3: Downloadservice in Google Chrome

Für den Downloadservice zeichnet das IKG der ETH Zürich verantwortlich. Der Datenzugriff über diesen Service ist browserbasiert, das heisst, die verfügbaren Geodatensätze können direkt im Browser dargestellt und (je nach Datensatz) auf einzelne Layer, eine bestimmte Auflösung oder einen bestimmten Zeitstand eingeschränkt werden. Der ausgewählte Ausschnitt kann für die weitere Verarbeitung im eigenen GIS-Programm heruntergeladen werden.

Für die Webservices von geodata4edu.ch zeichnet die HSR verantwortlich. Dieser Zugang bietet die verfügbaren Datensätze als Map-, Feature- oder ImageServices zur Einbindung ins eigene GIS-Programm an (ArcGIS, QGIS in Planung) und erlaubt so das direkte Arbeiten (Exploren, Darstellen, Extrahieren etc.) mit den Geodaten. Tauchen Fragen auf, kann sich die Nutzerin oder der Nutzer einer zugriffsberechtigten Hochschule direkt an das an der ETH-Bibliothek angesiedelte Helpdesk wenden. Hier wird entschieden, wie prioritär die Anfrage ist, ob sie selbst beantwortet wird, ob sie an einen der beiden Serviceverantwortlichen, also an die HSR oder das IKG, weitergegeben werden muss oder ob gar die Informatikdienste der am Service beteiligten Hochschulen zu kontaktieren sind. Der Nutzer wird in jedem Fall seine Antwort direkt vom Helpdesk der ETH-Bibliothek erhalten.

Die Website von geodata4edu.ch liefert umfassende Informationen zur Nutzung des Service, technische Informationen und einen Projektbeschrieb. Ein Schulungsangebot für die assoziierten Teilnehmer wird gegenwärtig an der ETHBibliothek aufgebaut. 


\section{Das Projekt}

\section{Ausgangslage}

Wie bereits erwähnt, besteht trotz steigender Nachfrage nach Geodaten in verschiedenen Wissenschaftszweigen bis heute keine zentrale akademische Geodateninfrastruktur in der Schweiz. Ein neuer Service sollte diese Lücke schliessen.

Um den Kontext des lancierten Projekts geodata4edu.ch verständlich $\mathrm{zu}$ machen, wird nachfolgend eine Auswahl bereits vorhandener wichtiger Dienste und Services, in deren Zentrum die Visualisierung und/oder der Zugang zu Geodaten stehen, kurz skizziert:

- Kartenportal. $\mathrm{CH}^{8}$ : Bei der von einem Konsortium von Kartensammlungen in Schweizer Bibliotheken entwickelten Webanwendung handelt es sich um einen raumbezogenen Kartenkatalog. Die räumliche Ausdehnung von in Schweizer Bibliotheken vorhandenen Karten kann auf einem Google-basierten Karteninterface angezeigt werden und es besteht eine Verbindung zum originalen Eintrag im Bibliothekskatalog.

- Atlas der Schweiz ${ }^{9}$ : Dieser Service wurde Mitte 2016 veröffentlicht. Die am IKG der ETH Zürich vollständig neu entwickelte Version des Nationalatlas ermöglicht die virtuelle Erforschung der Schweiz und die interaktive Visualisierung räumlicher und zeitlicher Zusammenhänge in neuen Dimensionen. Der Atlas bietet die dreidimensionale Visualisierung sämtlicher Daten sowie zeitlicher Abläufe auf einem virtuellen Globus. Die verwendeten Daten lassen sich in der entwickelten Applikation in einer qualitativ hochstehenden kartographischen Darstellung detailliert anzeigen und vergleichen. Die eigentlichen Daten aber bleiben dem Nutzenden für eine weitere Verwendung vorenthalten, denn die Exportmöglichkeit besteht aus Screenshots in unterschiedlichen Bild- und Dokumentformaten (jpg, png, pdf).

- geo.admin.ch ${ }^{10}$ : Dies ist die Plattform für geolokalisierte Informationen, Daten und Dienste der Bundesverwaltung. Die Daten werden von öffentlichen Einrichtungen zur Verfügung gestellt und via Internet auf geo.admin.ch öffentlich zugänglich gemacht. Über diesen Service kann u.a. auch auf die Landeskarte der Schweiz zugegriffen werden. Sie ist eine erste öffentliche Anwendung basierend auf der sich im Aufbau befindenden Nationalen Geo-

8 http://www.kartenportal.ch/. Vgl. hierzu auch den Beitrag „Kartenportal.CH“ von Jost SchmidLanter in diesem Band.

9 https://www.atlasderschweiz.ch/de/.

10 https://www.geo.admin.ch/de/home.html. 
dateninfrastruktur (NGDI) ${ }^{11}$ im Rahmen des Projektes e-geo.ch ${ }^{12}$. Der Service ermöglicht die Suche und reine Anzeige von geographischen Informationen. Es können aber auch hier keine originalen Geodaten heruntergeladen werden.

Bereits vor der Lancierung des Projektes geodata4edu.ch haben sich das Institut für Kartografie und Geoinformation der ETH Zürich sowie die Hochschule für Technik Rapperswil mehrere Jahre intensiver mit Geodaten beschäftigt. Eine wichtige Voraussetzung für umfassendere Aktivitäten mit Geodaten wurde mit dem Inkrafttreten des Geoinformationsgesetzes (GeoIG) ${ }^{13}$ im Jahr 2007 geschaffen, indem der Bezug von Geodaten für die Ausbildung und Forschung zumindest preiswerter und verbreitet möglich wurde. Denn neben den in der Regel frei zugänglichen national, kantonal und kommunal organisierten Geodatenabgabestellen sind für Lehre und Forschung noch weitere, meist kostenpflichtige, Geodaten von Belang.

Diese Ausgangslage war zum einen für das IKG im Jahr 2010 der Anstoss, die Applikation GeoVITe für die Visualisierung und den Bezug von individuell zusammengestellten Geodaten der swisstopo $\mathrm{zu}$ entwickeln. Die ETH-Bibliothek übernahm bereits damals die Aufgabe, das Angebot in den Räumlichkeiten der Bibliothek anzubieten und die Benutzerinnen und Benutzer bei Fragen rund um Geodaten kompetent zu beraten. Zum anderen wurde an der HSR mit vorwiegend nationalen und überkantonalen Projekten ein Kompetenzzentrum Geoinformation für den generellen Ausbildungsbetrieb geschaffen. Diese Fachstelle hat unter anderem die Aufgabe, die redundante Geodatenhaltung an der Fachhochschule $\mathrm{zu}$ verhindern und somit den personellen und finanziellen Aufwand zu minimieren.

Um Synergien optimal nutzen zu können und der Schweizer Wissenschaftscommunity einen umfassenden Service für diverse Geodatensets anbieten zu können, haben sich die HSR, das IKG sowie die ETH-Bibliothek bereits im Jahr 2014 darauf verständigt, ein Kooperationsprojekt zu lancieren. Im Rahmen dieses Vorhabens sollten die bereits etablierten Angebote der HSR und der ETH Zürich zusammengeführt und $\mathrm{zu}$ einem nationalen Service ausgebaut werden. Um Doppelspurigkeiten zu vermeiden, war es ein wichtiger Aspekt des Projektes,

11 Bundes Geodaten-Infrastruktur: https://www.geo.admin.ch/de/ueber-geo-admin/leistungsa uftrag/bundes-geodaten-infrastruktur.html.

$12 \mathrm{https} / / /$ www.geo.admin.ch/e-geo-ch.

13 Bundesgesetz über Geoinformation: http://www.admin.ch/opc/de/classified-compilation/20 050726/index.html. 
dass sich die neue Dienstleistung von anderen Angeboten in der Schweiz abgrenzt.

Die Zeit für die Lancierung eines Projektes war insofern günstig, als im Rahmen des Programms SUK P-2 „Wissenschaftliche Information: Zugang, Verarbeitung und Speicherung“ (2013-2016) von den Hochschulen finanzielle Mittel beantragt werden konnten. Unterstützt wurden Vorhaben für den Aufbau eines umfangreichen Grundangebotes an digitalen Inhalten von wissenschaftlicher Relevanz und optimale Werkzeuge für deren Verarbeitung für die Forschenden, Lehrenden und Lernenden.

Für die Realisierung eines Geodatenportals ergänzen sich die Fachkompetenzen der drei Projektpartner ideal: Das IKG zeichnet für technische und forschungsrelevante Aspekte verantwortlich, die HSR für die nutzerrelevanten sowie ebenfalls technischen Belange und die ETH-Bibliothek für die administrativen und prozessualen Aspekte, für die Aufgaben des Projektmanagements sowie für das zukünftige Marketing und das Schulungsangebot.

Die Projektpartner waren von Beginn an überzeugt, dass der neue nationale Service für Geodaten für die Schweizer Wissenschaftscommunity einen grossen Nutzen bieten würde. Sie haben das gemeinsame Ziel, diesen Service in der Hochschullandschaft der Schweiz zu etablieren und ihn unverzichtbar für das Auffinden und den Bezug von Geodaten zu machen.

\section{Antrag}

Ein erster gemeinsamer Projektantrag wurde von den genannten Partnern im Herbst 2014 eingereicht. Die erste Projektphase dauerte inklusive einer kostenneutralen Verlängerung von Januar 2015 bis Ende März 2017. Ein Folgeantrag im Rahmen des Programms P-5 „Wissenschaftliche Information“ (2017-2020) für die nahtlose Projektverlängerung für weitere knapp zwei Jahre folgte dann im Herbst 2016. Beide Anträge wurden vom Lenkungsausschuss der beiden Programme bewilligt und die finanziellen Mittel in Höhe von rund CHF 1,8 Mio. gesprochen. Ziel des ersten Projektantrages Geodata4SwissEDU war es somit, einen leistungsfähigen nationalen Service für das gezielte Auffinden, den Zugriff, die Präsentation, den Download und die Verarbeitung eines umfassenden Angebots an lizenzpflichtigen Geodaten von Bundesämtern und Kantonen für die Ausbildung und Forschung an Schweizer Hochschulen und Fachhochschulen aufzubauen. Der produktive Service hat mittlerweile den Namen ,geodata4edu.ch“ erhalten.

Ein zentraler nutzerfokussierter Zugang zu einer Vielzahl an Geodaten ist in mehrfacher Hinsicht effizient. So können etwa die Nutzerinnen und Nutzer die für sie relevanten Daten einfach und schnell auffinden. Darüber hinaus können Pro- 
jektdaten je nach Bedarf einer anderen Community zentral zur Verfügung gestellt werden. Zudem können beim Aufbau und Betrieb der technischen Infrastruktur sowie des Services Synergien zwischen den Projektpartnern effizient genutzt und dadurch Kosten gespart werden. Auf diese Weise können die einzelnen Hochschulen den Aufwand für die Bereitstellung von Geodaten massiv reduzieren. Das Modell kann etwa verglichen werden mit dem Service EDINA ${ }^{14}$ in Grossbritannien, der u.a. Geodaten für den landesweiten akademischen Bereich zur Verfügung stellt. EDINA ist an der Universität in Edinburgh lokalisiert.

Für den Aufbau eines nachhaltigen Service in der Schweiz haben die Projektpartner für die Realisierung von geodata4edu.ch folgende Grundsätze definiert:

- Nutzerfreundlicher zielgerichteter Zugriff für verschiedene Zielgruppen: Das Geodatenportal ist nutzerfreundlich, um das effiziente Auffinden und den erleichterten Zugang zu den angebotenen Daten zu garantieren. Der Service ist resultatorientiert und ermöglicht eine schnelle Beantwortung verschiedener Forschungsfragen. Die Dienstleistung spricht Forschende und Studierende mit unterschiedlichen Vorkenntnissen an, damit der Service sowohl von Personen mit GIS-Fachwissen als auch von jenen mit GIS-Grundwissen verwendet werden kann. Die Geodaten können je nach Verwendungszeck in einem Browser visualisiert oder in einem Desktop-GIS bearbeitet werden.

- Breites Datenangebot: Der Service bietet einen zentralen Zugang zu einem umfassenden Geodatenangebot, wodurch auch in dieser Hinsicht die Bedürfnisse von ganz unterschiedlichen Nutzerinnen und Nutzer abgedeckt werden. Im Angebot sind: ausgewählte offizielle Geodaten der swisstopo (in einer späteren Phase allenfalls auch Daten weiterer Bundesämter wie dem Bundesamt für Statistik oder Daten von Fachplanungen wie bspw. Raumplanung), ausgewählte offizielle Geodaten der Kantone und Gemeinden sowie spezielle anfallende Projektdaten, die auch für andere Nutzer von Interesse sind.

- Effizientes Datenmanagement: Die zentrale Infrastruktur des Geodatenportals ermöglicht die nationale Bündelung von Ressourcen und Kompetenzen für die Speicherung und das effiziente Management der Daten. Um die Qualität sicherzustellen, werden die Daten auf Vollständigkeit geprüft. Hierzu gehört ebenfalls die zentrale Abwicklung der erforderlichen Lizenzverträge und damit auch in dieser Hinsicht eine wesentliche Vereinfachung für die Nutzerinnen und Nutzer bzw. die teilnehmenden Institutionen.

- Nachhaltiges Businessmodell und Marketing: Die Nachhaltigkeit des Projektes ist mit einem fundierten Businessmodell und einem professionellen Marketing garantiert. Für die möglichst selbsttragende und langfristige Finanzie-

14 www.edina.ac.uk/. 
rung des Services wird ein Kundenstamm aufgebaut. Die teilnehmenden Hochschulen müssen entsprechende finanzielle Beiträge leisten.

- Schulungsangebot: Die teilnehmenden Institutionen bzw. die Endnutzerinnen und Endnutzer profitieren von einem Supportangebot sowie von Schulungen bzw. Schulungsunterlagen. Durch Nutzerfeedback wird der Service kontinuierlich verbessert und noch intuitiver gestaltet.

\section{Umsetzung}

Nachdem im ersten Projektjahr die Konzepte für das neue nationale Portal für Geodaten erarbeitet wurden, stand das zweite Projektjahr im Zeichen der technischen Umsetzung. Der hohe personelle Einsatz im zweiten Projektjahr ermöglichte den erfolgreichen Aufbau der technischen und inhaltlichen Komponenten des neuen Service, so dass geodata4edu.ch am Ende der ersten Projektphase, d. h. Ende März 2017, betriebsbereit war.

Bei der Projektumsetzung musste aus technischer Sicht zunächst zumindest eine virtuelle Zusammenführung der beiden bestehenden Infrastrukturen und Zugriffstechnologien der ETH Zürich sowie der HSR realisiert und weiterentwickelt werden. Da es das Projektziel war und ist, den Service an möglichst vielen wissenschaftlichen Institutionen in der Schweiz einzuführen, musste er auch technisch für eine grössere Anzahl von Nutzerinnen und Nutzer ausgebaut werden und skalierbar sein. Auch die entsprechenden Betriebskonzepte waren zu entwickeln.

Für das gezielte und rasche Auffinden und für den Zugriff auf die an den beiden Hochschulen dezentral vorliegenden Geodaten wurde ein Zugang in Form einer Website in deutscher, französischer und englischer Sprache entwickelt. Für die Suche nach Datensätzen wurde eine Metadatenbank mit definiertem Datenschema aufgebaut. Dank einer Wortübersetzungsliste ist auch die mehrsprachige Suche möglich (D, F, E). Die Datenbank wurde über eine API-Schnittstelle in die zentrale Website integriert. 


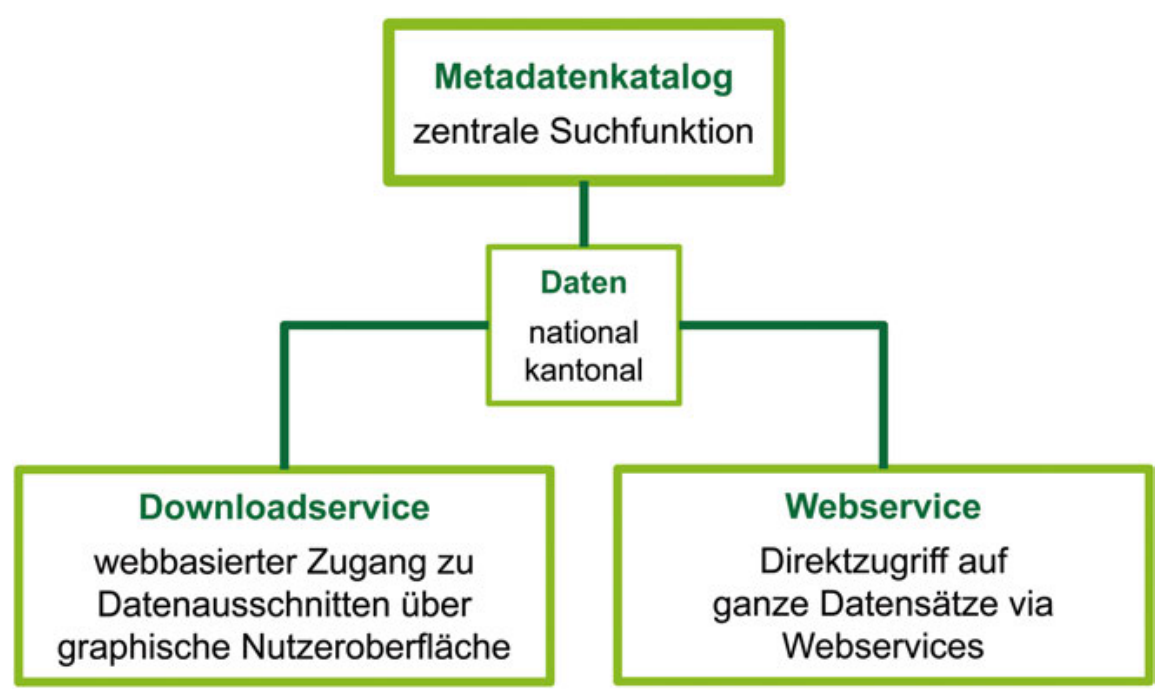

Abb. 4: Aufbau von geodata4edu.ch

Nutzerinnen und Nutzer mit keiner oder wenig GIS-Erfahrung können den sogenannten „Downloadservice“ (vgl. Abb. 4) nutzen. Zugang, Visualisierung und Download der Geodaten erfolgen hier über einen webbasierten Zugriff. Im ersten Projektjahr wurde die bereits vor dem Projekt bestehende Plattform GeoVITe des IKG auf Basis eines Usability-Tests überarbeitet und zu Beginn des zweiten Projektjahres erfolgreich auf der skalierbaren Infrastruktur der Informatikdienste der ETH Zürich neu aufgesetzt und implementiert sowie mit zusätzlichen Funktionen ausgestattet. Die Datensätze der swisstopo wurden und werden laufend für die Visualisierung aufbereitet; ebenso werden die Metadaten aus GeoVITe für die zentrale Metadatenbank im XML-Format gemäss vereinbartem Standard bereitgestellt.

Für Nutzerinnen und Nutzer mit GIS-Erfahrung wurden die sog. Webservices realisiert (vgl. Abb. 4). Im ersten Projektjahr wurde auf der Grundlage des Entscheids für eine Umsetzung des Zugangs mit der Variante Portal for ArcGIS mit Anbindung an SWITCHaai eine Testumgebung konzipiert, die Authentifizierung via SWITCHaai umgesetzt und die Zugänge zu den HSR-Services for Geodata aufgesetzt. Die Geodaten sind auf einer skalierbaren Infrastruktur von SWITCH gespeichert. Die Funktionen und die Performance der angebotenen Services wurden von internen und externen Nutzern getestet, entsprechend verbessert und weiter optimiert. Pilotkunde ist die Zürcher Hochschule für Angewandte Wissenschaften (ZHAW). Das Betriebskonzept wurde erarbeitet und wird basierend auf Erfahrungen aus dem laufenden Betrieb ergänzt. 
Rechtliche Aspekte und Datenqualität spielen für die Gewährleistung eines professionellen Betriebes des Geodatenportals eine zentrale Rolle und mussten entsprechend sorgfältig abgeklärt werden. Um auch für den Gesamtbetrieb die rechtlichen Rahmenbedingungen zu schaffen, wurde ein Kooperationsvertrag zwischen der HSR und der ETH Zürich abgeschlossen, der die Zusammenarbeit zwischen den Projektpartnern im Rahmen von geodata4edu.ch regelt. Weitere Verträge waren und sind zwischen den Lizenzgebern und dem Serviceanbieter auszuarbeiten wie auch die rechtlichen Rahmenbedingungen für die Verwendung der Daten durch die Hochschulen. Auch die Datennutzungsvereinbarungen zwischen den an geodata4edu.ch teilnehmenden Hochschulen sowie die Nutzungsbedingungen von geodata4edu.ch sind Teil des komplexen Vertragswerkes.

Zur Datenqualität kann seitens des Projektteams nur wenig beigetragen werden, da diese primär von den Datenlieferanten abhängig ist. Die Überprüfung auf Vollständigkeit wird jedoch vor Einspielung bzw. Publikation als Services durch die Projektpartner vorgenommen, allenfalls werden auch Anmerkungen zur Qualität in den Metadaten erfasst.

Das Businessmodell wurde auf Basis der zu erwartenden jährlichen Gesamtbetriebskosten von geodata4edu.ch erarbeitet. Beinhaltet sind Personal- und Infrastrukturkosten, Bereitstellungs- und Lizenzgebühren für Geodaten und Software sowie weitere Kosten. Der Betrieb soll nicht gewinnorientiert sein, Rückstellungen für minimale Weiterentwicklungen sind jedoch geplant. Für die Berechnung der jährlichen Zugriffsgebühren werden die einzelnen Hochschulen jeweils als eine Einheit betrachtet. Für eine Institution basiert die Höhe der Gebühren gegenwärtig auf der Anzahl der Studierenden, der Forschenden und des administrativen Personals. Im Verlauf der ersten Projektphase war es aus rein praktischen Gründen noch nicht möglich, einen ersten Kundenstamm aufzubauen. Es ist naheliegend, dass für eine Vermarktung ein funktionierender Service aufgebaut sein muss und die rechtlichen Rahmenbedingungen geklärt sein müssen. Längerfristiges Ziel ist es, mit den Einnahmen der assoziierten Teilnehmer einen möglichst kostendeckenden Betrieb von geodata4edu.ch sicherzustellen. Mit finanziellen Einnahmen von Seiten der assoziierten Teilnehmer kann aufgrund des Budgetierungsprozesses der Hochschulen allerdings kaum vor 2018 gerechnet werden. Dies bedeutet, dass für eine erste Betriebsphase von geodata4edu.ch derzeit noch Projektgelder investiert werden müssen.

Bereits während der ersten Projektphase konnte zumindest das Konzept des zukünftigen Services im Rahmen von diversen Anlässen ersten potenziellen Kundinnen und Kunden vorgestellt und es konnten Flyer in der Fachcommunity verteilt werden, so bspw. am 10. UNIGIS-Tag Schweiz an der HSR Rapperswil oder am ESRI TechDay auf dem Gurten in Bern. Einem breiten bibliothekarischen Fachpublikum wurde das Projekt auf der LIBER Konferenz 2016 (Konferenz Euro- 
päischer Forschungsbibliotheken) in Helsinki mit einer Poster-Präsentation vorgestellt. $^{15}$

\section{Erste Erfahrungen und Ausblick}

Zentraler Aspekt des gegenwärtig laufenden Folgeprojektes geodata4edu.ch ist es, den nationalen Service an Schweizer Hochschulen möglichst breit bekannt zu machen und die ersten Teilnehmer zu gewinnen. Der bereits erwähnte LaunchEvent im März 2017 war hierzu ein wichtiger Meilenstein. Seither konnte der Service an mehreren Hochschulen in allen Landesteilen der Schweiz präsentiert werden. Die Nachfrage nach weiteren Präsentationen ist ein Indiz dafür, dass an den einzelnen Institutionen ein potenzieller Bedarf für die neue Dienstleistung vorhanden ist. Bis Mitte 2017 haben eine Hochschule aus der Westschweiz und zwei Hochschulen aus der Deutschschweiz die erforderlichen Verträge für den Zugriff auf die Daten bis Ende 2018 unterzeichnet und sind damit ,assoziierte Teilnehmer' geworden. Weitere konkrete Anfragen von weiteren Hochschulen sind in Bearbeitung. Da eine erworbene Zugriffsberechtigung jeweils für die gesamte Institution Gültigkeit hat, stellt sich stets die Frage, welche Stelle die Verträge abschliesst und wie die Finanzierung geregelt wird. Wenn sich die jeweiligen Hochschulbibliotheken um die Erwerbung des Geodatenzugangs kümmern, können sie auf jeden Fall von den Erfahrungen mit der Lizenzierung von anderen Informationsressourcen profitieren.

Weitere Ziele für die zweite Projektphase bis Ende 2018 sind die Stärkung von geodata4edu.ch mit laufenden Optimierungen sowie Erweiterungen des Datenangebotes und der Funktionen. Dies wird die Attraktivität des Portals für Nutzerinnen und Nutzer steigern und eine nachhaltige Etablierung in der Schweizer Hochschullandschaft erleichtern. Wichtige Teilvorhaben sind etwa die Erweiterung des webbasierten Zugangs um themenspezifische Kollektionen, der Ausbau des Datenangebotes um weitere Datenherren oder die Integration historischer Daten. Das Usability Lab der Hochschule für Technik und Wirtschaft Chur hat im ersten Halbjahr 2017 einen Usability Test des Einstiegportals durchgeführt. Die ausgewerteten Ergebnisse werden der Optimierung des Einstiegsportals und des Metadatenkatalogs dienen. Für die Vermarktung des Services geodata4edu.ch ist ein einheitlicher Auftritt aller am Projekt beteiligten Parteien essentiell. Hierzu

15 Arlette Piguet, Anna Babel u.a.: Geodata for Swiss Education. Implementation of a national geoportal. Beitrag an der Postersession der LIBER Annual Conference. Helsinki, Finland, 29. Juni bis 01. Juli 2016 (https://doi.org/10.3929/ethz-a-010688568). 
wurde ein kleiner Leitfaden für die Kommunikation nach aussen erarbeitet. Wichtig wird auch sein, den einzelnen Hochschulen die Vorteile von geodata4edu.ch aufzuzeigen, u.a. die Minimierung des eigenen Aufwandes für das Auffinden und die Bereitstellung von Geodaten. Für gezielte Optimierungen ist auch die Auswertung der Benutzeranfragen unerlässlich. Damit können die FAQs auf der Website bedarfsgerecht ergänzt oder Unklarheiten in den Anleitungen der beiden Services behoben werden. Zusätzlich wird jede Anfrage in einer Excel-Tabelle mit einigen definierten Details erfasst. Diese Datensammlung wird die Grundlage sein für den zukünftigen Aufbau eines Ticketing-Systems für die professionelle Verwaltung der Benutzeranfragen. Aufgrund der besonderen Bedeutung von Geodaten für eine Vielzahl von Disziplinen ist es naheliegend, dass auch dem Aspekt Schulung von und für Studierende oder andere Nutzer im laufenden Betrieb hohe Aufmerksamkeit geschenkt werden muss. Wie bereits erwähnt, sind entsprechende Aktivitäten gegenwärtig im Aufbau.

Da bis Ende 2018 noch Projektgelder zur Verfügung stehen, können die Kosten für die einzelnen Hochschulen gegenwärtig auf einem niedrigen Niveau angesetzt werden. Aufgrund des aktuellen Businessplanes müssen die Beiträge ab 2019 für die assoziierten Teilnehmer allerdings massiv erhöht werden. Es stellt sich daher bereits heute die Frage, ob die einzelnen Institutionen in der Lage sein werden, den Service nachhaltig zu finanzieren. Ob in Zukunft mit einer permanenten zusätzlichen Finanzierung durch Fördergelder des Bundes gerechnet werden kann, ist heute noch offen. Inwieweit geodata4edu.ch weiter ausgebaut werden kann, hängt letztlich von den verfügbaren finanziellen Mitteln ab. Bei einem grossen Benutzeraufkommen muss der Service auf jeden Fall weiter skaliert werden, was die Kosten nochmals in die Höhe treibt. In Zukunft wäre sogar die Integration umfassender räumlicher Datensätze möglich. Solche Daten werden in den nächsten Jahren gerade im akademischen Bereich vermehrt anfallen, z.B. aus Forschungsprojekten mit raumbezogenen Fragestellungen und Resultaten.

Den Betreiberinnen von geodata4edu.ch ist bewusst, dass auch auf kantonaler oder auf Bundesebene analoge Infrastrukturen für umfassendere Angebote an Geodaten aufgebaut werden könnten. Ob solche Dienstleistungen dann auch die Bedürfnisse des akademischen Bereiches tatsächlich befriedigen könnten, wäre abzuwarten. Wenn es gelingt, geodata4edu.ch als nationalen Service an möglichst vielen Schweizer Hochschulen und Fachhochschulen zu etablieren und die nachhaltige Finanzierung zu sichern, wird der neue Service in Zukunft sicherlich eine unverzichtbare Informationsquelle für den Forschungsplatz Schweiz werden. Ein substantieller Beitrag für die Forschungsqualität an den Schweizer Hochschulen könnte damit geleistet werden. 
Bereitgestellt von | ETH-Bibliothek Zürich Angemeldet Heruntergeladen am | 22.08.18 14:22 